\title{
Phospholipids across scales: lipid patterns and plant development
}

Leia Axelle Colin and Yvon Jaillais

Laboratoire Reproduction et Développement des Plantes, Univ Lyon, ENS de Lyon, UCB Lyon 1, CNRS, INRA, F-69342 Lyon, France

Corresponding author: Yvon Jaillais (yvon.jaillais@ens-lyon.fr)

\begin{abstract}
:
Phospholipids are major building blocks of cell membranes and as such they have a key structural role in maintaining their integrity as a hydrophobic barrier. However, phospholipids not only have structural but also regulatory functions that are involved in a myriad of signaling pathways. Integrative approaches in plants recently revealed that certain phospholipids have distinct patterns of accumulation at the tissue or organ scales, which turned out to be important cues in a developmental context. Using examples on different phospholipid classes, including phosphatidylinositol-4,5-bisphosphate, phosphatidylserine, phosphatidylcholine and phosphatidic acid, we review how spatio-temporal lipid patterns arise at the organismal level and what are their downstream consequences on plant development.
\end{abstract}




\section{Abbreviations:}

PI4P, phosphatidylinositol-4-phosphate; $\mathrm{PI}(4,5) \mathrm{P} 2$, phosphatidylinositol-4,5-bisphosphate, PS, phosphatidylserine; PC, phosphatidylcholine; PE, phosphatidylethanolamine; PG, phosphatidylglycerol, DAG, diacylglycerol; PIPs, phosphoinositides; ER; endoplasmic reticulum; PM, plasma membrane; PH, pleckstrin homology; Lact, Lacthaderin; PLC,

Phospholipase C; PSS1, PHOSPHATIDYLSERINE SYNTHASE1; PIP5K, PHOSPHATIDYLINOSITOL-4-PHOSPHATE 5-KINASE; CVP, COTYLEDON VASCULAR PATTERN; CVL , CVP2-LIKE; ROP, RHO-OF-PLANTS; PECT, CTP:PHOSPHORYLETHANOLAMINE CYTIDYLYLTRANSFERASE; FT, FLOWERING LOCUS T; LHY, LONG ELONGATED HYPOCOTYL; CCA, CIRCADIAN CLOCK ASSOCIATED.

\section{Highlights:}

- $\mathrm{PI}(4,5) \mathrm{P}_{2}$ tissue-specific accumulation patterns control cell differentiation

- $\quad \mathrm{PI} 4 \mathrm{P} / \mathrm{PI} 4 \mathrm{~K} \beta \mathrm{s}$ accumulate at the cell plate of dividing cells and regulate cytokinesis

- PS variations at the tissue scale regulate membrane activities at the nanoscale

- Diurnal phospholipid oscillations regulate flowering and the circadian clock

\section{Graphical abstract:}

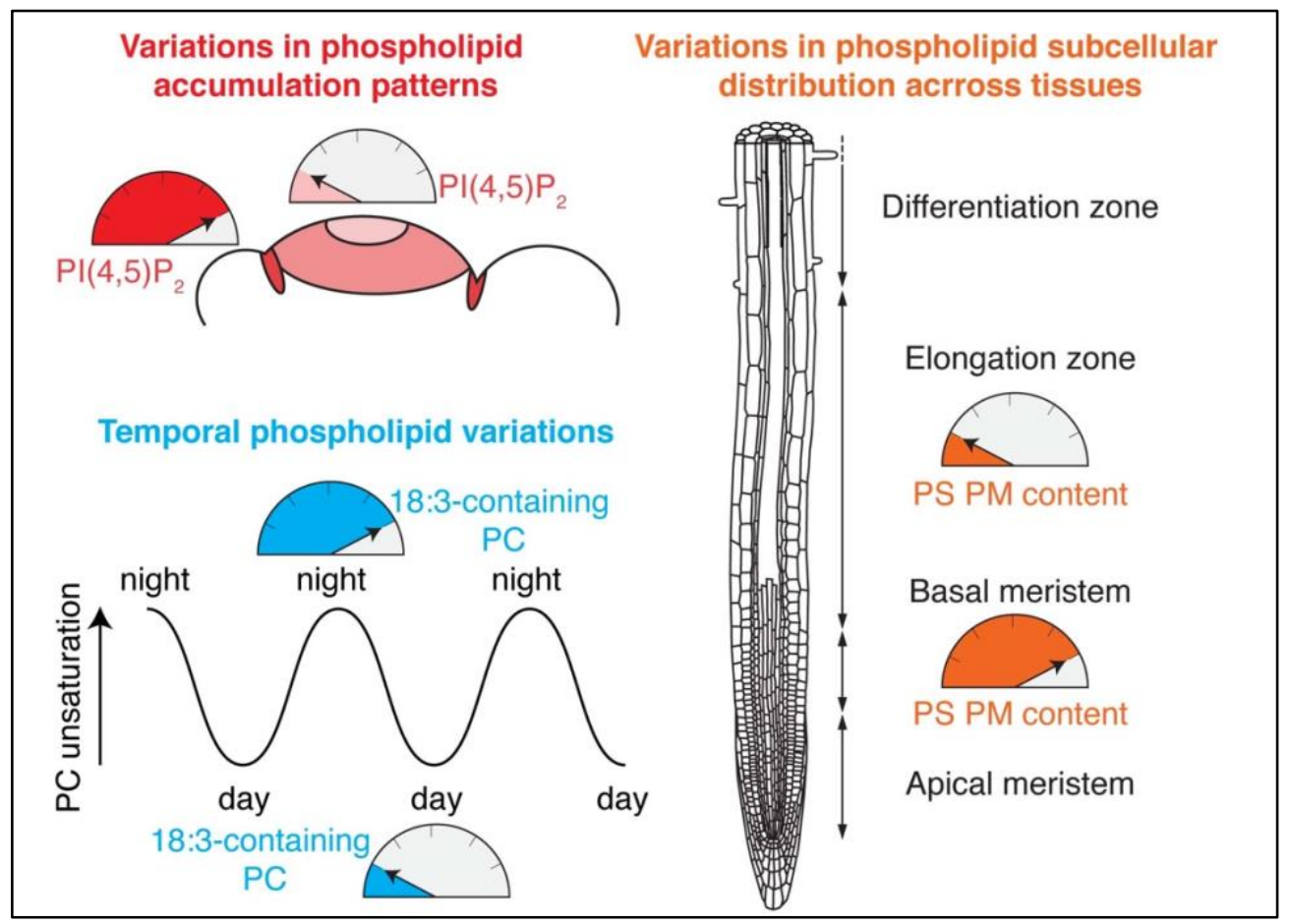




\section{Introduction}

Phospholipids, also referred to as glycerophospholipids, are a class of lipids whose structure is based on a glycerol backbone that connects a hydrophobic tail - consisting of two long-chain fatty acids linked at the $s n-1$ and $s n-2$ position (diacylglycerol) - and a hydrophilic head group (Figure 1a). Because of their amphiphilic nature, phospholipids are key structural components of membrane bilayers, with the fatty acid chains aggregating together in the core of the bilayer and the hydrophilic heads on both sides. The simplest phospholipid class, with only a phosphate group at position sn-3, is phosphatidic acid (PA). The phosphate head group can be further modified to form the different classes of phospholipids, by addition of a choline, serine, ethanolamine, inositol or glycerol molecule, forming phosphatidylcholine (PC), phosphatidylserine (PS), phosphatidylethanolamine (PE), phosphatidylinositol (PI) or phosphatidylglycerol (PG), respectively (Figure 1a). PG main functions are in plastids and mitochondria and will not be discussed hereafter [1]. Phosphatidylinositol can be further phosphorylated on the 3rd, 4th and/or 5th position of the inositol head group to form phosphoinositides (PIPs, also referred to as polyphosphoinositides) such as phosphatidylinositol-4-phosphate (PI4P) and phosphatidylinositol-4,5-bisphosphate $\left(\mathrm{PI}(4,5) \mathrm{P}_{2}\right)$ (Figure 1a) [2].

There are different ways to classify phospholipids based on for example: i) their relative abundance (abundant vs minor lipids, i.e. less than $5 \%$ of total phospholipids), ii) their net charges (neutral vs anionic), iii) their metabolic fluxes (relatively stable concentration vs acute production/interconversion and fast turn-over), iv) their site of production (produced at the site of action or exported), v) their shape (conical, straight or inverted conical) or vi) their fatty acid chains (number of carbons:number of unsaturation) (Figure 1a and b). Overall, abundant phospholipids (e.g. PC, PE) are often considered as "structural" lipids in the sense that they are essential to establish a hydrophobic barrier. These phospholipids are produced by biosynthetic enzymes mainly in the endoplasmic reticulum (Figure $1 \mathrm{~b}$ and c). Low abundant lipids (e.g. PA and PIPs) are labelled as "signaling" lipids in the sense that they mostly have regulatory effects on membrane functions. These signaling lipids are negatively charged, which enable specific interactions with proteins. In addition, they have fast turn-over and can be produced in an acute manner by lipid kinases, phosphatases or phospholipases at their site of action outside of the endoplasmic reticulum (Figure $1 \mathrm{~b}$ and $\mathrm{c}$ ) [2].

However, the frontiers between the "structural" and "signaling" lipids are not always clear-cut. Indeed, these categories mostly arise from considerations at the cellular scale, initially drawn from studies using single cell systems such as yeast or in vitro cell culture. As such, 
phospholipid biosynthesis and localization are often depicted in a single cell. However, recent evidence suggests that specific pattern of phospholipid accumulation arise at the tissue or organ level in plants, particularly for classical "signaling" lipids but also for "structural" lipids. These patterns have downstream consequences on cell behaviors ultimately impacting plant development. Here, we review three examples in which spatiotemporal phospholipid pattern impacts plant development in different ways, highlighting the importance of integrative approaches to study phospholipid biology.

\section{Phosphatidylinositol-4,5-bisphosphate patterns and their roles in tissue differentiation}

Phosphoinositides are minor phospholipids, making less than $1 \%$ of total phospholipids (Figure 1a). They have specific accumulation patterns in the different compartments of the cell and as such they determine organelle identity [2,3]. Furthermore, because of their respective charges and shape, they are key determinants of membrane physicochemical parameters (Figure 1b) [2]. Some of these parameters appears to be invariant between different tissues. For example, in all cell types analyzed so far, the plasma membrane is the most electronegative compartment, a feature that specifies its identity [4]. The strong electrostatic field of the plasma membrane is powered by the accumulation of PI4P in this membrane, the most abundant phosphoinositide species in plants (Figure 1a) [4]. PI4P accumulation at the plasma membrane appears to be invariant when analyzing different tissue and organ [4]. However, during cell division, PI4P strongly accumulates at the cell plate, which is therefore highly electrostatics [4][5]. The accumulation of PI4P at the cell plate correlates with the localization of PI4K $\beta 1$ in this organelle and the double mutant pi4k $\beta 1 \beta 2$ is defective in cytokinesis and phragmoplast establishment $[6,7]$. By contrast, $\mathrm{PI}(4,5) \mathrm{P}_{2}$ is not only patterned at the level of the cell but also at the tissue/organ scales.

Evidence for $\mathrm{PI}(4,5) \mathrm{P}_{2}$ patterning at the tissue scale comes from quantitative imaging studies of $\mathrm{PI}(4,5) \mathrm{P}_{2}$ sensors lines [8]. These transgenic lines stably express $\mathrm{PI}(4,5) \mathrm{P}_{2}$ binding domains fused to a fluorescent protein as an indirect way to localize this phospholipid [9-12]. Indeed, such fusion proteins are recruited to membranes that accumulate this lipid [12]. By contrast, in the absence of its target lipid, the sensor accumulates in the cytoplasm as a default localization [12]. In some cases, the chronic absence of the biosensor cognate lipid induces its subsequent destabilization and degradation. For example, the $\mathrm{C} 2$ domain of the bovine Lacthaderin protein (C2Lact), a PS-binding domain, is unstable and degraded when expressed in a mutant background that lacks PS biosynthesis [13]. Quantitative 3-D imaging revealed that the $\mathrm{PI}(4,5) \mathrm{P}$-binding Plekstrin Homology (PH) domain of Phospholipase C (PHPLC) accumulates 
to a low extent at the center of the shoot apical meristem in Arabidopsis, while it is stabilized in the boundary region (Figure 2a) [8]. By analogy to the C2Lact sensor, one may speculate that destabilization of PHpLc occurs when PI(4,5) $\mathrm{P}_{2}$ levels are low. This hypothesis was recently substantiated in cotyledons, as PHPLC accumulates about four fold when seedling are treated with a high concentration of $\mathrm{NaCl}$ (a condition known to induce $\mathrm{PI}(4,5) \mathrm{P}_{2}$ synthesis [10]) or $\mathrm{U}$ 73122 (which inhibits PI(4,5)P2 hydrolysis by phosphoinositide-specific-PLC (PI-PLC) and thereby promotes its accumulation) [14]. Although the exact role of the $\mathrm{PI}(4,5) \mathrm{P}_{2}$ pattern in the shoot apical meristem needs to be defined, it could be involved in the two main functions of the meristem: stem cell maintenance and organogenesis. For instance, in the clv3-17 mutant, which displays a larger central zone with more stem cells, the $\mathrm{PI}(4,5) \mathrm{P}_{2}$ pattern tends to disappear, suggesting that destabilization of the PHPLC sensor correlates with stem cell maintenance [8]. The shoot apical meristem is also characterized by a typical mechanical stress pattern, with strong and highly anisotropic tensions in boundaries compared to the central zone (Figure 2a) [15]. A positive correlation between the accumulation of $\mathrm{PI}(4,5) \mathrm{P}_{2}$ sensors and mechanical stresses in the boundary zones suggests that the $\mathrm{PI}(4,5) \mathrm{P}_{2}$ pattern could be linked with tissue mechanics and mechanical signal transduction [8]. Future work will reveal how this $\mathrm{PI}(4,5) \mathrm{P}_{2}$ pattern is established and what its function is in shoot meristem biology.

Lipid sensors are not the only clues pointing at the importance of specific $\mathrm{PI}(4,5) \mathrm{P} 2$ patterns at the tissue level. Indeed, PI4P 5-kinases (PIP5K), PI(4,5)P2 5-phosphatases and PI-PLCs have tissue specific expression patterns [16-21]. For example, PIP5K1, PIP5K2, PLC3 and PLC5 are expressed in the root meristem and in vascular tissues and PIP5K2 expression is regulated by the phytohormone auxin [16-18][19,20]. Furthermore, the plasma membrane-localized 5phosphatase COTYLEDON VASCULAR PATTERN2 (CVP2) is expressed in developing vascular cells in various organs and displays exquisite expression specificity for the protophloem in the root [22-25]. CVP2 and its homolog CVP2-LIKE1 (CVL1), as well as PIP5K1 and PIP5K2 are involved in venation pattern establishment in cotyledons, while plc2, plc3 and plc5 mutants have root architecture phenotypes [17,19,20,22]. The cvp2 cvll double mutant root protophloem fails to fully differentiate, as revealed by the presence of undifferentiated "gap" cells within protophloem strands (Figure 2b) [25]. Surprisingly, CVP2 overexpression, which promotes $\mathrm{PI}(4,5) \mathrm{P}_{2}$ processing into $\mathrm{PI} 4 \mathrm{P}$, also induces protophloem gap cells and phenocopies the cvp2 cvll double mutant, which accumulates $\mathrm{PI}(4,5) \mathrm{P}_{2}$ (Figure 2b) [25]. Furthermore, inducible overexpression at the plasma membrane of a highly processive PIP5K, which boosts $\mathrm{PI}(4,5) \mathrm{P}_{2}$ production at the expense of the PI4P pool, also induces gap cells in developing protophloem (Figure 2b) [24]. Therefore, it appears that the PI(4,5) $\mathrm{P}_{2} / \mathrm{PI} 4 \mathrm{P}$ 
balance, specifically in protophloem cells, rather than the absolute amount of $\mathrm{PI}(4,5) \mathrm{P}_{2}$ is critical for the correct differentiation of this tissue (Figure $2 b$ ).

Cellular analyses revealed a correlation between increased vacuolar trafficking upon the alteration of the $\mathrm{PI}(4,5) \mathrm{P}_{2} / \mathrm{PI} 4 \mathrm{P}$ ratio and protophloem differentiation, suggesting that vacuole biogenesis and/or related endocytic trafficking regulated by these phosphoinositides could be linked with phloem development [24]. Furthermore, PIP5K1 and PIP5K2 are not only localized at the plasma membrane but also in the nucleus $[17,18]$. Nuclear targeting is actively regulated, at least in the case of PIP5K2 [26]. These localizations suggest that $\mathrm{PI}(4,5) \mathrm{P}_{2}$ could also have a direct role in gene expression regulation, which has been reported in animal systems [27,28]. Overall, these data indicate that the establishment of specific $\mathrm{PI}(4,5) \mathrm{P}_{2}$ accumulation pattern at the tissue level are hallmarks of tissue differentiation, at least in some cases. Some of the future challenges will be to sort which of the pleiotropic functions of this signaling lipids could be instrumental to regulate tissue differentiation.

\section{Phosphatidylserine variations at the tissue level regulate plasma membrane organization at the nanoscale}

PS shares characteristics with both above-mentioned phospholipid categories. Similar to "structural" phospholipids, it is synthesized in the endoplasmic reticulum by a single enzyme called PHOSPHATIDYLSERINE SYNTHASE 1 (PSS1) in Arabidopsis and PS is not acutely produced in compartments outside of the endoplasmic reticulum (Figure 1b and c) [29]. However, it is acidic, interacts with specific proteins and is present at low levels (i.e. from 1 to $5 \%$ of total phospholipids depending on the tissue [29]), which is a hallmark of "signaling" lipids (Figure 1a and b) [2]. PS in produced in the luminal leaflet of the endoplasmic reticulum and is then actively orientated by flippases in the cytoplasmic membrane leaflet [30]. This regulation is organelle specific, which allows the accumulation of PS in the cytoplasmic leaflet of post-Golgi compartments [30]. Localization studies using PS specific biosensors revealed that it accumulates at the plasma membrane and all along the endocytic pathway in Arabidopsis $[4,13]$. This accumulation gradient is critical to define an "electrostatic" membrane territory within the cell, which specifies the identity of plasma membrane-derived organelles [13]. Interestingly, the relative accumulation of PS biosensors at the plasma membrane or endosomes varies during root epidermis differentiation (Figure 3a) [31]. Indeed, PS biosensors mostly accumulate at the plasma membrane in meristematic cells, while they partition more evenly between the cell surface and endosomes in differentiating epidermal cells (Figure 3a). Experimental manipulation of the PS content by knock-out, knock-down or overexpression of 
the Arabidopsis PSS1 gene leads to the graded activation of plasma membrane auxin signaling pathway, which is regulated by the small GTPase RHO-OF-PLANTS6 (ROP6) [31,32]. Interestingly, PS is dispensable for the proper targeting of ROP6 to the plasma membrane [31]. However, super resolution microscopy and single molecule imaging revealed that PS is required for the auxin-induced clustering and stabilization of ROP6 in plasma membrane nanodomains (Figure $3 b$ and $c$ ). ROP6 stabilization in nanoclusters is required for downstream auxin signaling. This effect is dose-dependent, with no stabilization of ROP6 in nanodomains in the absence of PS and therefore no downstream signaling, even when ROP6 is constitutively active (Figure 3c). By contrast, ROP6 is hyper stabilized in nanodomains in response to auxin in PSS1 overexpressing plants (Figure 3c). Accordingly, these plants have the same phenotypes as plants expressing a constitutive active version of ROP6, including the chronic inhibition of endocytosis and a hypergravitropic root response [31].

One of the future challenges will be to decipher how is the PS pattern developmentallycontrolled during cell differentiation, a process which could involve lipid flippases or regulation of PS transfer at membrane contact sites, which are close (below 30nm) and stable apposition of two membranes involves, among other functions, in lipid exchanges [30,33-36]. Furthermore, PS itself is present in plasma membrane nanodomains, and single molecule tracking experiments revealed that these PS molecules are slow moving within the plane of the plasma membrane [31]. This raises the question of how a phospholipid may be immobilized within a membrane and whether this process could also be regulated at the tissue scale.

\section{Temporal phospholipid patterns}

The last example is perhaps the least expected as it entails PC, an archetype "structural" lipid. Indeed, transgenic lines with imbalanced PE/PC ratio, obtained by inducible knock-down or overexpression of CTP:PHOSPHORYLETHANOLAMINE CYTIDYLYLTRANSFERASE1 (PECT1, a rate limiting enzyme in PE biosynthesis), revealed that PC levels at the shoot apex correlate with flowering time: lines with heightened PC content flower early, while plants with reduced PC levels are late flowering (Figure 4a) [37,38]. The early flowering time phenotype observed in plants with heightened PC content is dependent upon expression of FLOWERING LOCUS T (FT), a master regulator of flowering time [39]. Interestingly, FT directly binds to PC but not PE in vitro [37]. Furthermore, PC but not PE oscillate depending of the time of day, with specific molecular species of PC showing various temporal pattern of accumulation (Figure $4 b$ ). For example, PC species containing less unsaturated fatty acids are predominant 
during the day, while PC species with linolenic acid (18:3) increase during the night (Figure 4b). In vitro, PC species with linolenic acid bind poorly to FT by contrast to less unsaturated PC species [37]. Transgenic plants that overexpress FATTY ACID DESATURASE3 (FAD3) show high content in PC species with 18:3 fatty acids and are late flowering (Figure 4b) [37,40]. Together these data suggest that FT preferentially interacts with the PC species that accumulate during the day to promote flowering (Figure 4b) [37]. However, it remains unclear how PC contributes to FT activity at the molecular level and how FT discriminates the different PC species based on their degree of unsaturation.

Of note, PC is not the only phospholipid showing temporal pattern of accumulation; and some molecular species of PA or PS also cycle diurnally [41]. These cycling are not just regulated by the light/dark cycle but they are under direct circadian regulation and are abolished in clock mutants [42]. Interestingly, PA itself regulates the core clock components LONG ELONGATED HYPOCOTYL (LHY) and CIRCADIAN CLOCK ASSOCIATED1 (CCA1). PA directly binds to both LHY and CCA1, which inhibits their DNA binding capacity [42]. Together these results suggest that the interaction of PA with LHY/CCA1 may function in a negative feedback loop to integrate the circadian clock with lipid metabolism (Figure 4c).

\section{Concluding remarks and future perspectives}

The examples highlighted in this review show that phospholipid patterns are multiscales, from membrane nanodomains up to the organismal scale, with specific phospholipid patterns arising according to the cell type, the tissue differentiation status or the time of day. Tissue-specific control of lipid patterns is an emerging concept, but its exploration is somewhat limited by our ability to monitor their dynamics and crosstalks. By design, genetically encoded phospholipid biosensors reveal lipid subcellular localization, but not their respective accumulation [12]. As such, these biosensors are suited to detect changes in phospholipid localization, as exemplified for PS [31]. However, if the levels of phospholipids vary but not their subcellular localization, the current tools are not quantitative enough to measure these variations with spatial resolution. In some cases, the stability of the lipid sensor is dependent on the quantity of this lipid within the cell. However, we do not understand the mechanistic bases for such lipid-induced stabilization/destabilization. We therefore urgently need new methods to image and quantify phospholipid accumulation and turnover with tissue and cellular resolution. Such methods could include for example the design of new quantitative lipid sensors, the use of lipid immunolocalization or mass spectrometry imaging of lipid species. The latter is particularly promising since it can not only discriminate different phospholipids but also their various 
unsaturation degree [43-46]. However, this technique is still limited to very abundant phospholipids, such as PC. In addition, the best resolution reported in plant samples to date is $\sim 10 \mu \mathrm{m}$, which is sufficient for analyses at the organ/tissue scales but not cellular/subcellular levels [43-46].

While we begin to uncover some of the enzymes involved in lipid patterning at the tissue scale, the next challenge will be to decipher what are the downstream targets that respond to these patterns, and what are the developmental consequences. This is a particularly daunting task since phospholipids have pleiotropic targets and are therefore expected to affect the localization or activity of many effector proteins. Pinpointing relevant interactions will therefore require in depth biochemical knowledge of the protein/lipid binding interfaces in order to modulate these interactions both from the lipid but also protein sides. At the same time, such efforts will require integrative biology approaches to blend deep biochemical knowledge into their cellular and developmental contexts.

\section{Acknowledgements.}

We thank the L. Armengot, P. Moreau, O. Hamant, M. Platre and T. Stanislas for comments on the manuscript, Y.J. is funded by the French National Research Agency ANR caLIPSO (ANR18-CE13-0025-02) and ANR STAYING-TIGHT (ANR-18-CE13-0016-02), L.C. is funded by a $\mathrm{PhD}$ fellowships from the French Ministry of Higher Education. 


\section{References}

1. Nakamura Y: Plant Phospholipid Diversity: Emerging Functions in Metabolism and Protein-Lipid Interactions. Trends Plant Sci 2017, 22:1027-1040.

2. Noack LC, Jaillais Y: Precision targeting by phosphoinoistides: how PIs direct endomembrane trafficking in plants. . Current Opinion in Plant Biology 2017, 40.

3. Boutte Y, Moreau P: Modulation of endomembranes morphodynamics in the secretory/retrograde pathways depends on lipid diversity. Curr Opin Plant Biol 2014, 22:22-29.

4. Simon ML, Platre MP, Marques-Bueno MM, Armengot L, Stanislas T, Bayle V, Caillaud MC, Jaillais Y: A PtdIns(4)P-driven electrostatic field controls cell membrane identity and signalling in plants. Nat Plants 2016, 2:16089.

5. Caillaud MC: Anionic Lipids: A Pipeline Connecting Key Players of Plant Cell Division. Front Plant Sci 2019, 10:419.

•• 6. Lin F, Krishnamoorthy P, Schubert V, Hause G, Heilmann M, Heilmann I: A dual role for cell plate-associated PI4Kbeta in endocytosis and phragmoplast dynamics during plant somatic cytokinesis. EMBO J 2019.

This paper shows that PI $4 \mathrm{~K} \beta 1$ localizes at the cell plate in dividing cells and that the growth phenotype of the pi $4 \mathrm{k} \beta 1$ pi $4 \mathrm{k} \beta 2$ double mutant is rescued when $\mathrm{PI} 4 \mathrm{~K} \beta 1$ is expressed under the control of the KNOLLE promoter, which is specific of late cytokinesis. PI $4 \mathrm{~K} \beta$ s are involved in the trafficking of PIN2, KNOLLE and clatrhin at the cell plate. In addition, the pi4k $\beta 1$ pi $4 \mathrm{k} \beta 2$ double mutant shows overstabilization of phragmoplast microtubules and mislocalization of MICROTUBULE-ASSOCIATED65-3 (MAP65-3), a phenotype that PI4K $\beta$ s regulate in close association with its binding partner, the MAP kinase MPK4.

7. Kang BH, Nielsen E, Preuss ML, Mastronarde D, Staehelin LA: Electron tomography of RabA4b- and PI-4Kbeta1-labeled trans Golgi network compartments in Arabidopsis. Traffic 2011, 12:313-329.

• 8. Stanislas T, Platre MP, Liu M, Rambaud-Lavigne LES, Jaillais Y, Hamant O: A phosphoinositide map at the shoot apical meristem in Arabidopsis thaliana. $B M C$ Biol 2018, 16:20.

Using quantitative live imaging of geneticaly encoded fluorescent lipid biosensors, this study reveals the existence of a stereotypical pattern of $\mathrm{PI}(4,5) \mathrm{P}_{2}$ accumulation at the shoot apical meristem, suggesting a possible morphogenetic action of this lipid in the shoot apex.

9. Simon ML, Platre MP, Assil S, van Wijk R, Chen WY, Chory J, Dreux M, Munnik T, Jaillais Y: A multi-colour/multi-affinity marker set to visualize phosphoinositide dynamics in Arabidopsis. Plant $J$ 2014, 77:322-337.

10. van Leeuwen W, Vermeer JE, Gadella TW, Jr., Munnik T: Visualization of phosphatidylinositol 4,5-bisphosphate in the plasma membrane of suspensioncultured tobacco BY-2 cells and whole Arabidopsis seedlings. Plant J 2007, 52:1014-1026.

11. Vincent P, Chua M, Nogue F, Fairbrother A, Mekeel H, Xu Y, Allen N, Bibikova TN, Gilroy S, Bankaitis VA: A Sec14p-nodulin domain phosphatidylinositol transfer protein polarizes membrane growth of Arabidopsis thaliana root hairs. $J$ Cell Biol 2005, 168:801-812.

12. Platre MP, Jaillais Y: Guidelines for the Use of Protein Domains in Acidic Phospholipid Imaging. Methods Mol Biol 2016, 1376:175-194. 
- 13. Platre MP, Noack LC, Doumane M, Bayle V, Simon MLA, Maneta-Peyret L, Fouillen L, Stanislas T, Armengot L, Pejchar P, et al.: A Combinatorial Lipid Code Shapes the Electrostatic Landscape of Plant Endomembranes. Dev Cell 2018, 45:465-480 e411.

Using a collection of phospholipid sensors and membrane surface charge probes, this study shows that there is an electrostatic gradient along the plant endocytic pathway that is set up by specific combinations of the anionic lipids PI4P, PA and PS.

- 14. Lee E, Vanneste S, Perez-Sancho J, Benitez-Fuente F, Strelau M, Macho AP, Botella MA, Friml J, Rosado A: Ionic stress enhances ER-PM connectivity via phosphoinositide-associated SYT1 contact site expansion in Arabidopsis. Proc Natl Acad Sci U S A 2019.

Using $\mathrm{PI}(4,5) \mathrm{P}_{2}$ biosensors, this study shows that $\mathrm{PI}(4,5) \mathrm{P}_{2}$ content increases upon ionic stress, which in turn recruits synaptotagmins (SYTs) and remodels endoplasmic reticulum/plasma membrane contact sites.

15. Hamant O, Heisler MG, Jonsson H, Krupinski P, Uyttewaal M, Bokov P, Corson F, Sahlin P, Boudaoud A, Meyerowitz EM, et al.: Developmental patterning by mechanical signals in Arabidopsis. Science 2008, 322:1650-1655.

16. Mei Y, Jia WJ, Chu YJ, Xue HW: Arabidopsis phosphatidylinositol monophosphate 5kinase 2 is involved in root gravitropism through regulation of polar auxin transport by affecting the cycling of PIN proteins. Cell Res 2012, 22:581-597.

17. Tejos R, Sauer M, Vanneste S, Palacios-Gomez M, Li H, Heilmann M, van Wijk R, Vermeer JE, Heilmann I, Munnik T, et al.: Bipolar Plasma Membrane Distribution of Phosphoinositides and Their Requirement for Auxin-Mediated Cell Polarity and Patterning in Arabidopsis. Plant Cell 2014, 26:2114-2128.

18. Ischebeck T, Werner S, Krishnamoorthy P, Lerche J, Meijon M, Stenzel I, Lofke C, Wiessner T, Im YJ, Perera IY, et al.: Phosphatidylinositol 4,5-bisphosphate influences PIN polarization by controlling clathrin-mediated membrane trafficking in Arabidopsis. Plant Cell 2013, 25:4894-4911.

19. Zhang Q, van Wijk R, Zarza X, Shahbaz M, van Hooren M, Guardia A, Scuffi D, GarciaMata C, Van den Ende W, Hoffmann-Benning S, et al.: Knock-Down of Arabidopsis PLC5 Reduces Primary Root Growth and Secondary Root Formation While Overexpression Improves Drought Tolerance and Causes Stunted Root Hair Growth. Plant Cell Physiol 2018, 59:2004-2019.

20. Zhang Q, van Wijk R, Shahbaz M, Roels W, Schooten BV, Vermeer JEM, Zarza X, Guardia A, Scuffi D, Garcia-Mata C, et al.: Arabidopsis Phospholipase C3 is Involved in Lateral Root Initiation and ABA Responses in Seed Germination and Stomatal Closure. Plant Cell Physiol 2018, 59:469-486.

21. Kanehara K, Yu CY, Cho Y, Cheong WF, Torta F, Shui G, Wenk MR, Nakamura Y: Arabidopsis AtPLC2 Is a Primary Phosphoinositide-Specific Phospholipase $C$ in Phosphoinositide Metabolism and the Endoplasmic Reticulum Stress Response. PLoS Genet 2015, 11:e1005511.

22. Carland FM, Nelson T: Cotyledon vascular pattern2-mediated inositol $(\mathbf{1 , 4 , 5 )}$ triphosphate signal transduction is essential for closed venation patterns of Arabidopsis foliar organs. Plant Cell 2004, 16:1263-1275.

23. Rodriguez-Villalon A, Gujas B, Kang YH, Breda AS, Cattaneo P, Depuydt S, Hardtke CS: Molecular genetic framework for protophloem formation. Proc Natl Acad Sci U S A 2014, 111:11551-11556. 
•• 24. Gujas B, Cruz TMD, Kastanaki E, Vermeer JEM, Munnik T, Rodriguez-Villalon A:

Perturbing phosphoinositide homeostasis oppositely affects vascular

differentiation in Arabidopsis thaliana roots. Development 2017, 144:3578-3589.

This work, together with Ref 25 , shows that the $\mathrm{PI}(4,5) \mathrm{P}_{2} / \mathrm{PI} 4 \mathrm{P}$ balance, as regulated by plasma membrane localized phosphatases and kinases, is critical for the differentiation of the root protophloem. Using various genetic background with impaired $\mathrm{PI}(4,5) \mathrm{P}_{2} / \mathrm{PI} 4 \mathrm{P}$ balance, this study establishes that both an increase or a decrease of the $\mathrm{PI}(4,5) \mathrm{P}_{2}$ content in protophloem induce the production of undiferentiated gap cells in protophloem tracks. Furthemore, it shows that a skewed $\mathrm{PI}(4,5) \mathrm{P}_{2} / \mathrm{PI} 4 \mathrm{P}$ ratio impact vacuolar trafficking, which could in turn impact phloem diffrentiation.

25. Rodriguez-Villalon A, Gujas B, van Wijk R, Munnik T, Hardtke CS: Primary root protophloem differentiation requires balanced phosphatidylinositol-4,5biphosphate levels and systemically affects root branching. Development 2015, 142:1437-1446.

- 26. Gerth K, Lin F, Daamen F, Menzel W, Heinrich F, Heilmann M: Arabidopsis phosphatidylinositol 4-phosphate 5-kinase 2 contains a functional nuclear localization sequence and interacts with alpha-importins. Plant $J$ 2017, 92:862878.

This work shows that PIP5K2 is not only localized at the plasma membrane but also in the nucleus. PIP5K2 is actively addressed to the nucleus by a functional nuclear localization signal, and interacts with alpha-importin. Furthermore, using immunolocalization, this study shows that both $\mathrm{PI} 4 \mathrm{P}$ and $\mathrm{PI}(4,5) \mathrm{P}_{2}$, which are the substrate and product of PIP5K2, respectively, are presents in the nucleus, more specicaly the nucleoplasm. Together, this work open the possibility that some of the phenotypes attributed to $\mathrm{PI}(4,5) \mathrm{P}_{2}$ may be due to gene expression regulation rather than membrane-related events.

27. Hamann BL, Blind RD: Nuclear phosphoinositide regulation of chromatin. $J$ Cell Physiol 2018, 233:107-123.

28. Bryant JM, Blind RD: Signaling through non-membrane nuclear phosphoinositide binding proteins in human health and disease. J Lipid Res 2019, 60:299-311.

29. Yamaoka Y, Yu Y, Mizoi J, Fujiki Y, Saito K, Nishijima M, Lee Y, Nishida I: PHOSPHATIDYLSERINE SYNTHASE1 is required for microspore development in Arabidopsis thaliana. Plant J 2011, 67:648-661.

30. Nintemann SJ, Palmgren M, Lopez-Marques RL: Catch You on the Flip Side: A Critical Review of Flippase Mutant Phenotypes. Trends Plant Sci 2019, 24:468478.

•• 31. Platre MP, Bayle V, Armengot L, Bareille J, Marques-Bueno MDM, Creff A, ManetaPeyret L, Fiche JB, Nollmann M, Miege C, et al.: Developmental control of plant Rho GTPase nano-organization by the lipid phosphatidylserine. Science 2019, 364:57-62.

Using live super-resolution single molecule imaging, single particle tracking, genetics and biochemistry, this study shows that PS acts as a developmentally-controlled lipid rheostat that regulates cellular auxin sensitivity and plant development. Variations of PS localization during root epidermis differentiation impact on the formation Rho GTPase nanoclusters at the plasma membrane, which are required to transduce the auxin signal at the cell surface.

32. Armengot L, Marques-Bueno MM, Jaillais Y: Regulation of polar auxin transport by protein and lipid kinases. $J$ Exp Bot 2016, 67:4015-4037. 
33. Chung J, Torta F, Masai K, Lucast L, Czapla H, Tanner LB, Narayanaswamy P, Wenk MR, Nakatsu F, De Camilli P: PI4P/phosphatidylserine countertransport at ORP5and ORP8-mediated ER-plasma membrane contacts. Science 2015, 349:428-432.

34. Moser von Filseck J, Copic A, Delfosse V, Vanni S, Jackson CL, Bourguet W, Drin G: Phosphatidylserine transport by ORP/Osh proteins is driven by phosphatidylinositol 4-phosphate. Science 2015, 349:432-436.

35. Sohn M, Korzeniowski M, Zewe JP, Wills RC, Hammond GRV, Humpolickova J, Vrzal L, Chalupska D, Veverka V, Fairn GD, et al.: PI(4,5)P2 controls plasma membrane PI4P and PS levels via ORP5/8 recruitment to ER-PM contact sites. $J$ Cell Biol 2018, 217:1797-1813.

36. Wang Y, Mousley CJ, Lete MG, Bankaitis VA: An equal opportunity collaboration between lipid metabolism and proteins in the control of membrane trafficking in the trans-Golgi and endosomal systems. Curr Opin Cell Biol 2019, 59:58-72.

37. Nakamura Y, Andres F, Kanehara K, Liu YC, Dormann P, Coupland G: Arabidopsis florigen FT binds to diurnally oscillating phospholipids that accelerate flowering. Nat Commun 2014, 5:3553.

38. Nakamura Y: Membrane Lipid Oscillation: An Emerging System of Molecular Dynamics in the Plant Membrane. Plant Cell Physiol 2018, 59:441-447.

39. Matsoukas IG: Florigens and antiflorigens: a molecular genetic understanding. Essays Biochem 2015, 58:133-149.

40. Arondel V, Lemieux B, Hwang I, Gibson S, Goodman HM, Somerville CR: Map-based cloning of a gene controlling omega-3 fatty acid desaturation in Arabidopsis. Science 1992, 258:1353-1355.

41. Maatta S, Scheu B, Roth MR, Tamura P, Li M, Williams TD, Wang X, Welti R: Levels of Arabidopsis thaliana Leaf Phosphatidic Acids, Phosphatidylserines, and Most Trienoate-Containing Polar Lipid Molecular Species Increase during the Dark Period of the Diurnal Cycle. Front Plant Sci 2012, 3:49.

•• 42. Kim SC, Nusinow DA, Sorkin ML, Pruneda-Paz J, Wang X: Interaction and Regulation Between Lipid Mediator Phosphatidic Acid and Circadian Clock Regulators. Plant Cell 2019, 31:399-416.

This paper shows that PA species cycle diurnaly and that PA production is dependent upon the core circadian regulators LHY and CCA 1 . In turn, PA directly binds to LHY and CCA 1 transcription factors, which inhibits their ability to interact with the promoter of their target genes, thereby inhibiting their activity, including promotion of PA production. Together, this study shows that PA participates in a negative feedback loop with the core circadian regulators LHY and CCA 1.

- 43. Woodfield HK, Sturtevant D, Borisjuk L, Munz E, Guschina IA, Chapman K, Harwood JL: Spatial and Temporal Mapping of Key Lipid Species in Brassica napus Seeds. Plant Physiol 2017, 173:1998-2009.

This study and refs 44 to 46 reports on the use of matrix assisted laser desorption/ionizationmass spectrometry imaging (MALDI-MSI) to map phospholipid species with a resolution of up to $10 \mu \mathrm{m}$. This technique shows heterogenous pattern of accumulation of PC species in seeds of various plants.

44. Lu S, Sturtevant D, Aziz M, Jin C, Li Q, Chapman KD, Guo L: Spatial analysis of lipid metabolites and expressed genes reveals tissue-specific heterogeneity of lipid metabolism in high- and low-oil Brassica napus L. seeds. Plant J 2018, 94:915932.

45. Sturtevant D, Duenas ME, Lee YJ, Chapman KD: Three-dimensional visualization of membrane phospholipid distributions in Arabidopsis thaliana seeds: A spatial 
perspective of molecular heterogeneity. Biochim Biophys Acta Mol Cell Biol Lipids 2017, 1862:268-281.

46. Sturtevant D, Lee YJ, Chapman KD: Matrix assisted laser desorption/ionization-mass spectrometry imaging (MALDI-MSI) for direct visualization of plant metabolites in situ. Curr Opin Biotechnol 2016, 37:53-60.

47. Welti R, Li W, Li M, Sang Y, Biesiada H, Zhou HE, Rajashekar CB, Williams TD, Wang $\mathrm{X}$ : Profiling membrane lipids in plant stress responses. Role of phospholipase $\mathrm{D}$ alpha in freezing-induced lipid changes in Arabidopsis. J Biol Chem 2002, 277:31994-32002. 


\section{Figures}

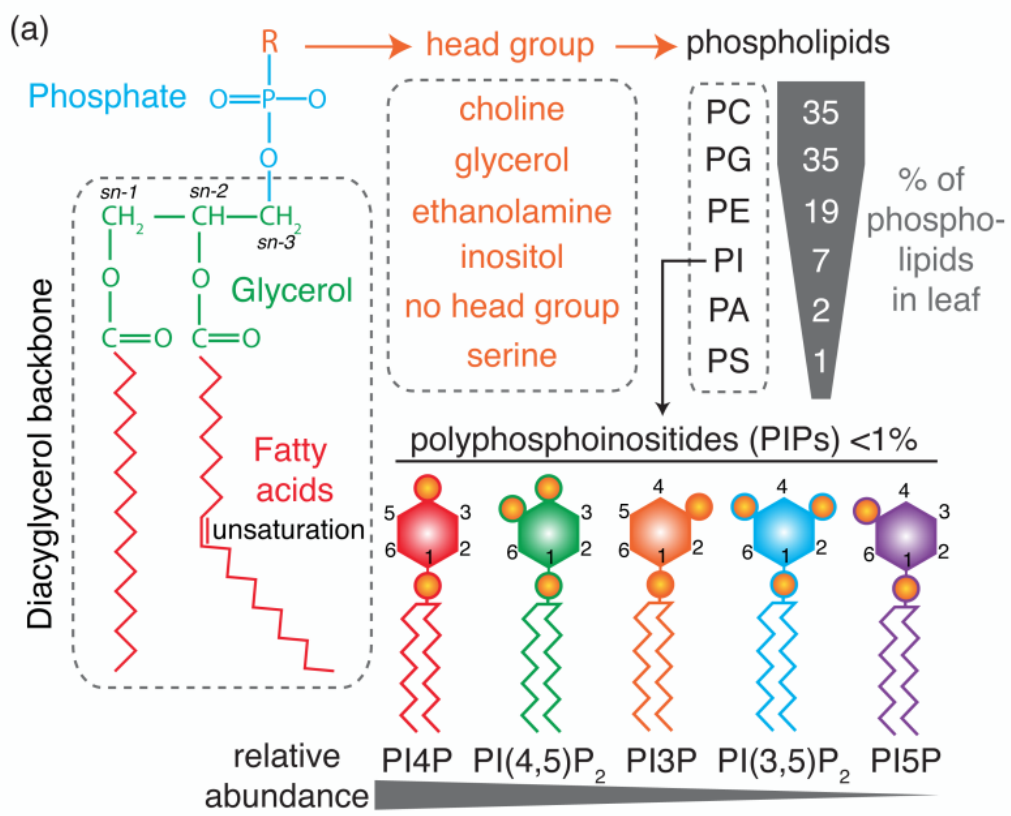

(b)

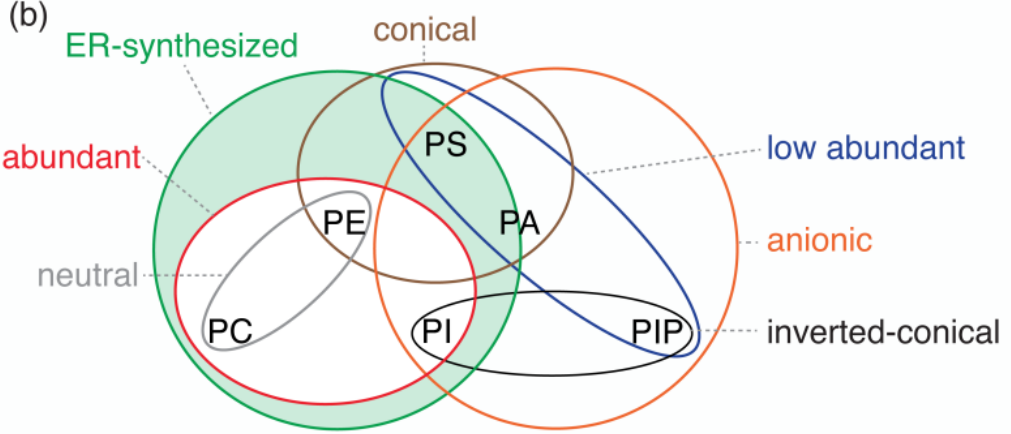

(c)

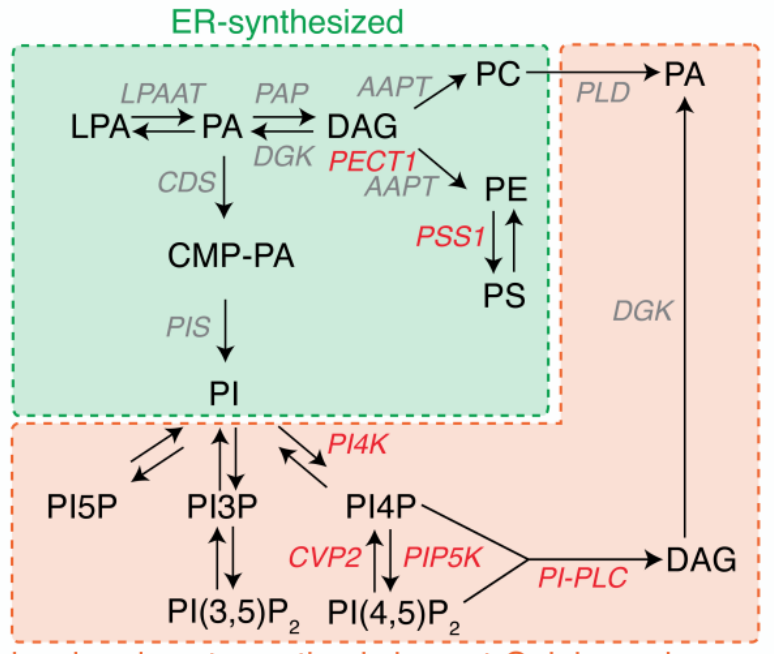

local and acute synthesis in post-Golgi membranes

Figure 1: Phospholipids properties and biosynthetic pathways. (a) Schematic representation of phospholipid general structure, with the relative amount of each phospholipid species depicted as measured in Arabidopsis leaf (data compiled from [29,47]). (b) Euler diagram illustrating phospholipid properties. (c) Phospholipid biosynthesis pathway. Note that phospholipid synthesis pathway in plastid/mitochondria are not depicted. The main rate- 
limiting enzymes involved in phospholipid interconversion are indicated (enzymes in red are discussed in this review). Abbreviations: DAG, diacylglycerol; PA, phosphatidic acid; PC, phosphatidylcholine; PE, phosphatidylethanolamine; PG, phosphatidylglycerol; PS, phosphatidylserine; PI, phosphatidylinositol; PI3P, phosphatidylinositol-3-phosphate; PI4P, phosphatidylinositol-4-phosphate; PI5P, phosphatidylinositol-5-phosphate; PI(3,5) $\mathrm{P}_{2}$, phosphatidylinositol-(3,5)-bisphosphate; $\mathrm{PI}(4,5) \mathrm{P}_{2}$, phosphatidylinositol-(4,5)-bisphosphate; LPAAT, LYSOPHOSPHATIDIC ACID ACYLTRANSFERASE; PAP, PA PHOSPHATASE; AAPT, AMINOALCOHOL AMINOPHOSPHOTRANSFERASE; DGK, DAG KINASE; PECT, CYTIDINE TRIPHOSPHATE:PHOSPHORYLETHANOLAMINE CYTIDYLYLTRANSFERASE; PSS, PS SYNTHASE; CDS, CYTIDINE DIPHOSPHATEDAG SYNTHASE; CMP-PA, CYTIDINE MONOPHOSPHATE PA; PIS, PI SYNTHASE; PI4K, PI4-KINASE; PIP5K, PI4P 5-KINASE; PLC, PHOSPHOLIPASE C; PLD, PHOSPHOLIPASE D. 
(a)

shoot apical meristem organization
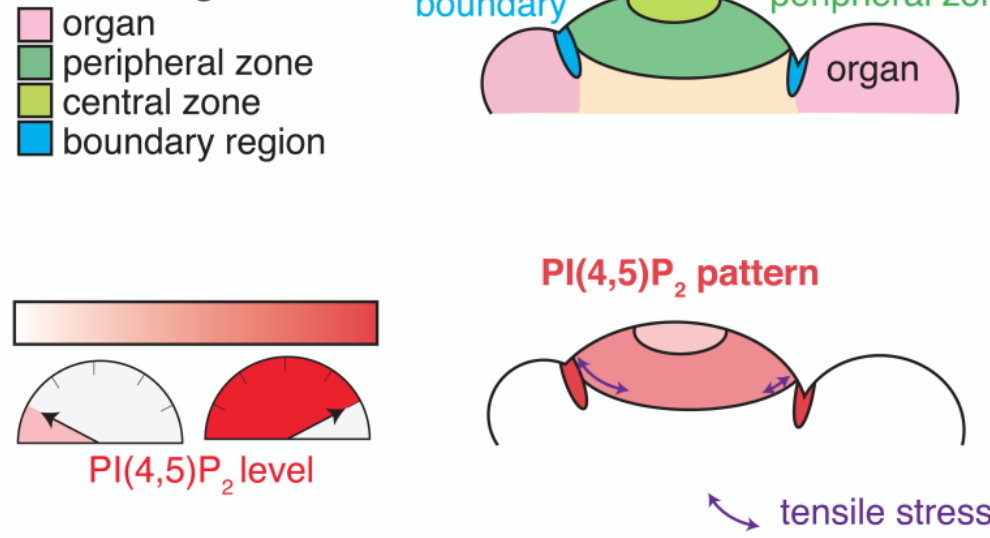
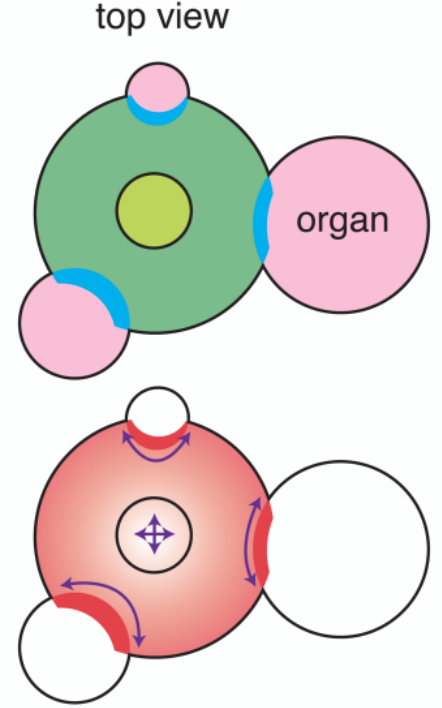

(b)

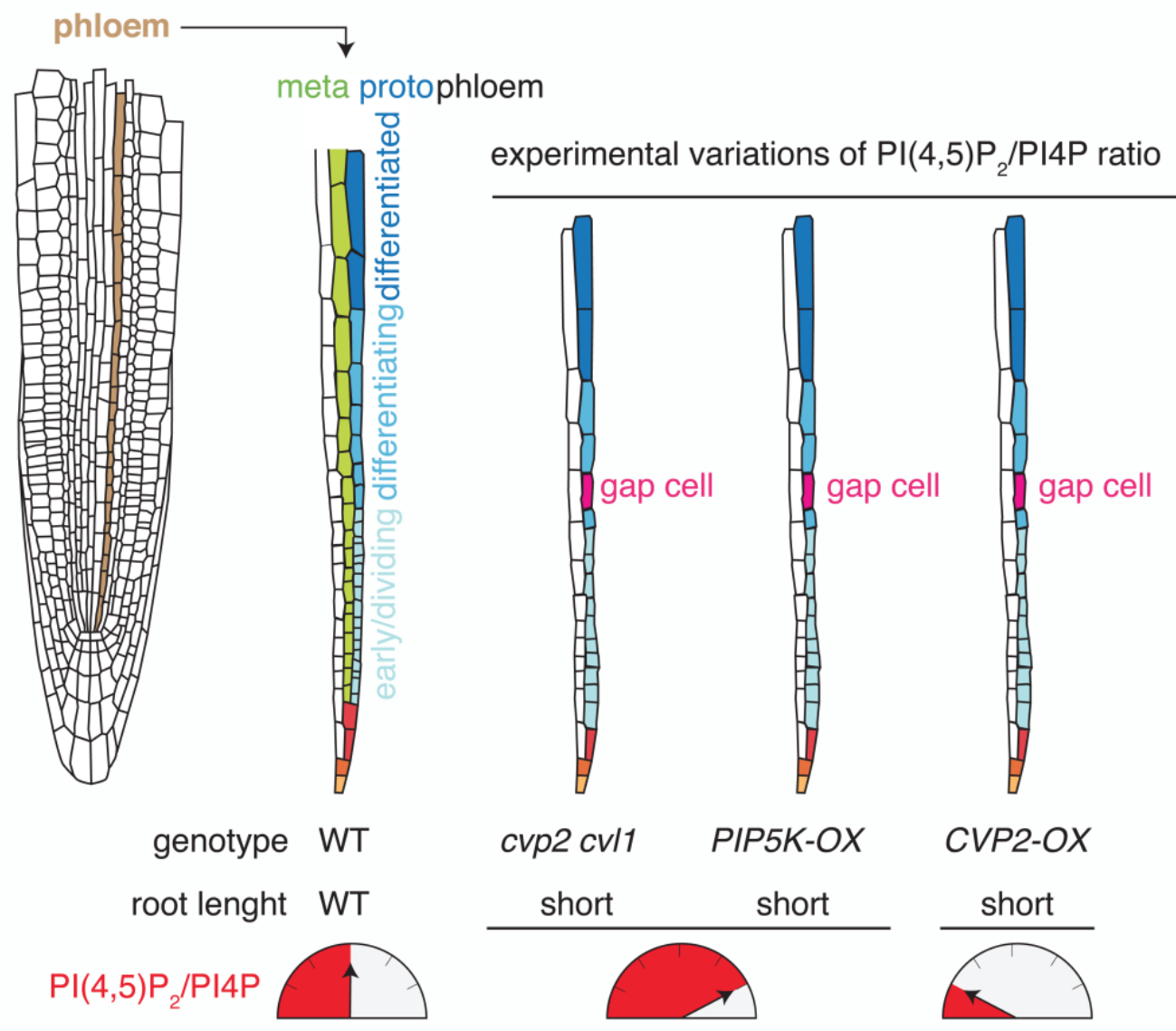

Figure 2: $\mathrm{PI}(4,5) \mathrm{P}_{2}$ patterning in meristematic tissues and impact of imbalanced $\mathbf{P I}(4,5) \mathrm{P}_{2} / \mathrm{PI} 4 \mathrm{P}$ ratio on protophloem differentiation. (a) Schematic representation of the shoot apical meristem organization (top) and $\mathrm{PI}(4,5) \mathrm{P}_{2}$ accumulation pattern together with the predicted tensile stress pattern (bottom). (b) Schematic representation of protophloem differentiation in Arabidopsis root meristem and phenotype associated with experimental variations in the $\mathrm{PI}(4,5) \mathrm{P}_{2} / \mathrm{PI} 4 \mathrm{P}$ balance. Root schematic was modified from B. Peret: https://figshare.com/articles/Primary_and_lateral_root_ai/5143987 
(a)

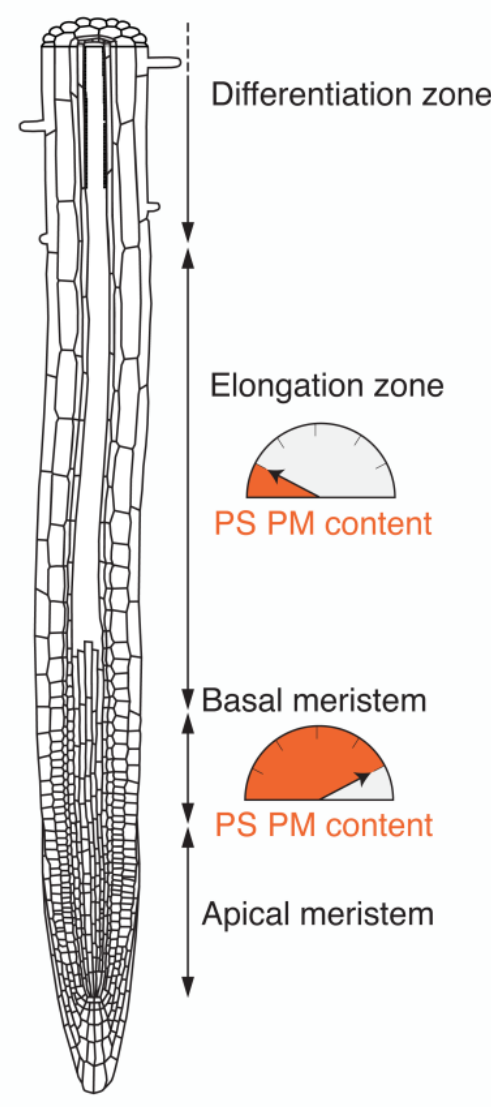

(b)

no auxin

PS nanoplateform (50-70 $\mathrm{nm}$ )

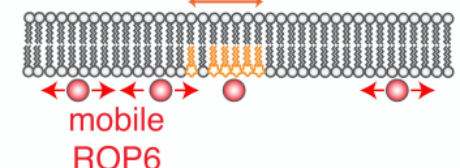

ROP6 minutes following auxin

PS nanoplateform $(50-70 \mathrm{~nm})$

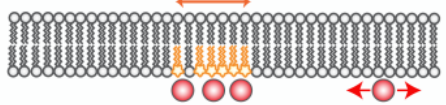

immobile

ROP6

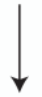

signaling

inhibition of endocytosis

microtubule orientation

gravitropism regulation

(c)

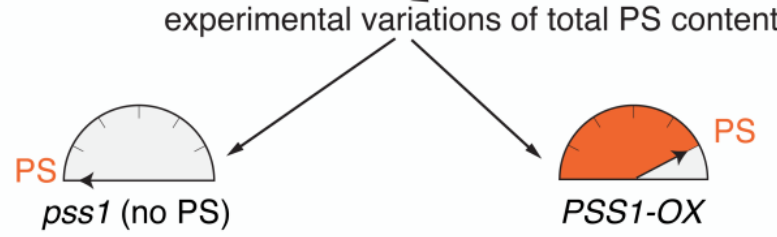

minutes following auxin

minutes following auxin

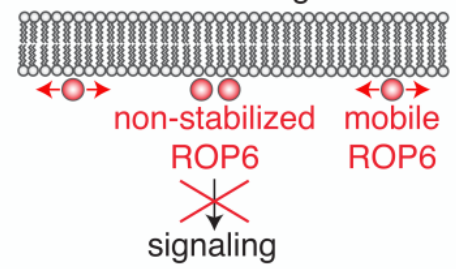

mimmic rop6 (loss-of-function)

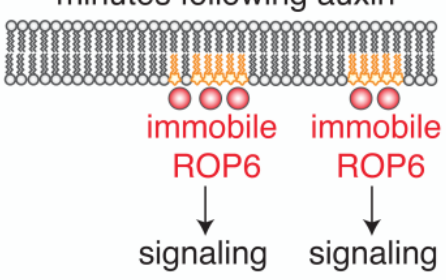

mimmic $R O P G^{C A}$

(gain-of-function)

Figure 3: Impact of PS variations on ROP6-mediated auxin signaling. (a) Variation of PS content at the plasma membrane during root differentiation. (b) Stabilization of ROP6 into PScontaining nanodomains upon auxin treatment and (c) impact of experimental variations of total PS content on ROP6 nanoclustering and downstream auxin signaling. Root schematic was modified from B. Peret: https://figshare.com/articles/Primary_and_lateral_root_ai/5143987 
(a)

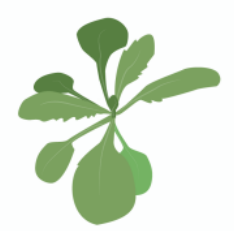

early flowering

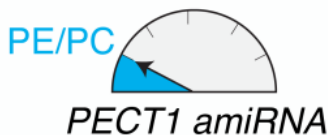

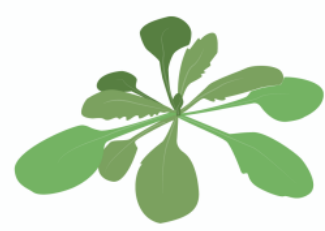

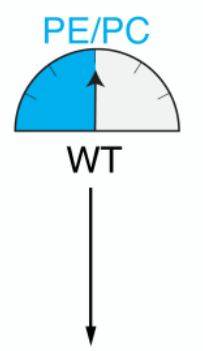

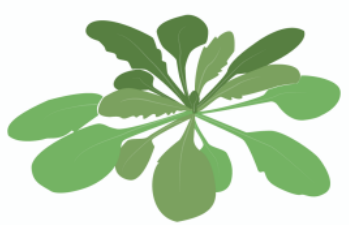

late flowering
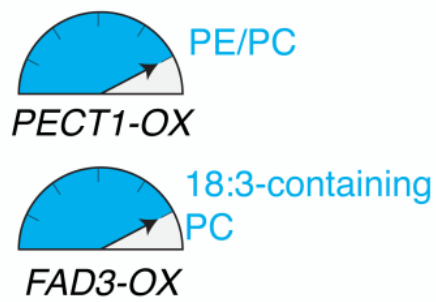

(b)

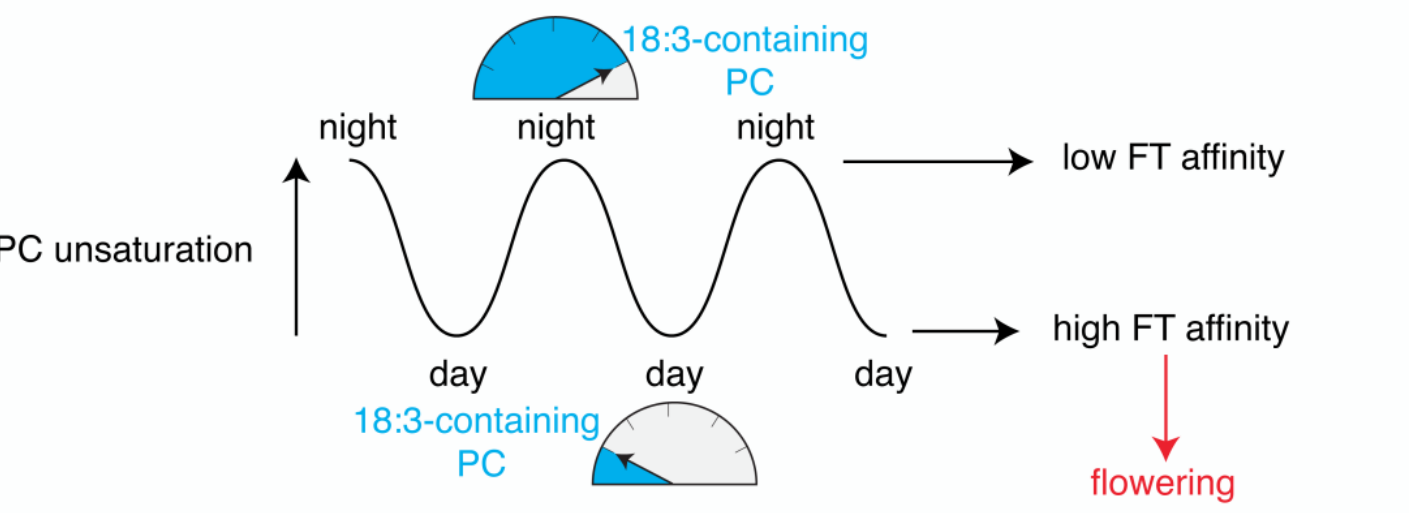

(c)

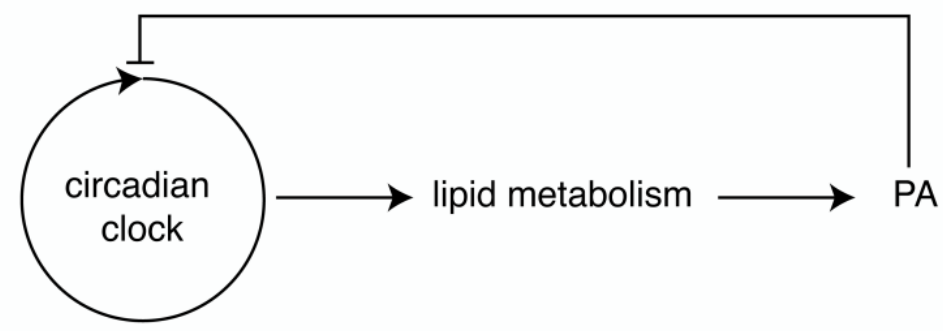

Figure 4: Impact of PC variations on flowering time and of PA on circadian clock. (a) Impact of experimental variations of the PE/PC ratio on flowering time and (b) diurnal variations of polyunsaturated 18:3-containing PC. (c) Feedback regulations between the circadian clock and PA. Arabidopsis rosette schematic was modified from F. Bouché: https://figshare.com/articles/Arabidopsis_-_Rosette_drawing_steps/4688839 